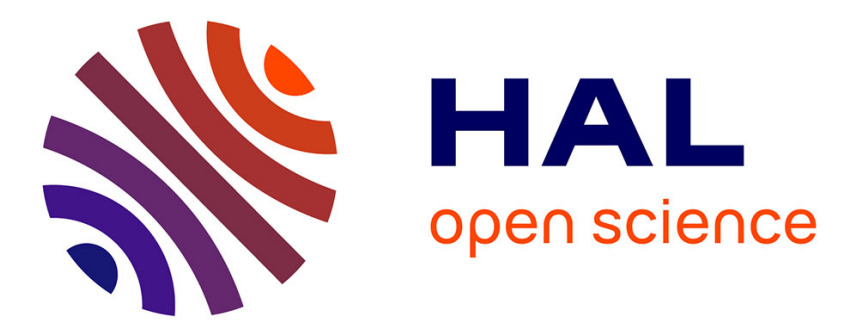

\title{
Observation of spectral drift in engineered quadratic nonlinear media
}

\author{
M. Marangoni, G. Sanna, D. Brida, Matteo Conforti, G. Cirmi, C. Manzoni, \\ F. Baronio, P. Bassi, C. de Angelis, G. Cerullo
}

\section{- To cite this version:}

M. Marangoni, G. Sanna, D. Brida, Matteo Conforti, G. Cirmi, et al.. Observation of spectral drift in engineered quadratic nonlinear media. Applied Physics Letters, 2008, 93 (2), pp.021107. 10.1063/1.2955529 . hal-02397760

\section{HAL Id: hal-02397760 \\ https://hal.science/hal-02397760}

Submitted on 6 Dec 2019

HAL is a multi-disciplinary open access archive for the deposit and dissemination of scientific research documents, whether they are published or not. The documents may come from teaching and research institutions in France or abroad, or from public or private research centers.
L'archive ouverte pluridisciplinaire HAL, est destinée au dépôt et à la diffusion de documents scientifiques de niveau recherche, publiés ou non, émanant des établissements d'enseignement et de recherche français ou étrangers, des laboratoires publics ou privés. 


\title{
Observation of spectral drift in engineered quadratic nonlinear media
}

\author{
M. Marangoni ${ }^{1, a)}$, G. Sanna ${ }^{2}$, D. Brida ${ }^{1}$, M. Conforti ${ }^{3}$, G. Cirmi ${ }^{1}$, C. Manzoni ${ }^{1}$, F. Baronio ${ }^{3}$, \\ P. Bassi ${ }^{2}$ C. De Angelis ${ }^{3}$, and G. Cerullo ${ }^{1}$
}

${ }^{1}$ ULTRAS-CNR-INFM, Dipartimento di Fisica, Politecnico di Milano

Piazza L. Da Vinci 32, I-20133 Milan, Italy.

${ }^{2}$ Dipartimento di Elettronica Informatica e Sistemistica, Università di Bologna,

Viale Risorgimento 2, I-40136 Bologna, Italy

${ }^{3}$ Dipartimento di Elettronica per l'Automazione, Università di Brescia

Viale Branze 38, I-25123 Brescia, Italy

\begin{abstract}
Spectral blue-shifts of femtosecond pulses induced by quadratic cascaded nonlinearities are experimentally studied in an engineered aperiodically-poled stoichiometric lithium tantalate crystal with optimized nonlinear pattern and chirp of the input pump pulses. Starting from nearly gaussian input pulses centred at $1420 \mathrm{~nm}$ with $70 \mathrm{~nm}$ spectral width, spectral shifts as high as $40 \mathrm{~nm}$ were obtained in combination with almost unaltered spectral shape and transform-limited pulse duration.
\end{abstract}

a) Corresponding author: tel. +39 02 23996070, fax +39 0223996126,

e-mail: marco.marangoni@polimi.it 

Cascaded processes in quadratic materials ${ }^{1}$ provide a very efficient tool for manipulation of the spectral and temporal properties of femtosecond pulses. Most attention has been devoted in the past few years to the temporal domain, and, in particular, to effects such as soliton propagation ${ }^{2}$, pulse compression $^{3,4}$, group velocity control $^{5}$ and, more recently, self-steepening ${ }^{6,7}$. One of the most intriguing spectral counterparts of these effects is the spectral shift that the fundamental frequency (FF) field undergoes in a second harmonic $(\mathrm{SH})$ generation process under suitable phase mismatch conditions and in the presence of strong group-velocity-mismatch (GVM) between the interacting waves $^{8,9}$. Such shift can be described in the framework of a quadratic self-steepening effect ${ }^{10}$, due to the asymmetric deformation of the FF temporal envelope when dragged by a slower temporally trapped SH pulse. A full control of quadratic spectral shifts, in sign, magnitude and shape, could be of great interest for the realization of "frequency shifting" devices, which would give tunability to fixed frequency femtosecond sources, such as mode-locked $\mathrm{Yb}$ and Er-doped fiber lasers.

Despite the large body of theoretical work, so far only few experimental studies on quadratic spectral shifts are available ${ }^{9,10,}$, performed under the conditions of constant phase mismatch and nearly transform-limited input pulses. Such studies highlight several limitations of the process: i) the output spectrum is deformed with respect to its original bell-shape, and its center of mass is much less shifted than its peak due to the formation of undesired tails; ii) the spectral shift occurs in the first few millimetres of propagation and rapidly saturates upon increasing the propagation distance; iii) the output energy of the FF pulses is lower than the input one due to strong pump depletion. To overcome some of these limitations, the use of engineered aperiodically-poled nonlinear crystals has been proposed and theoretically studied ${ }^{11,12}$. Such structures allow to locally tailor the nonlinear interaction between FF and SH pulses during propagation and have the potential of tuning the carrier frequency and of moulding the spectral characteristics of the FF pulses.

In this paper we experimentally show how spectral shifts of the FF pulses can be optimized by a proper combination of engineered aperiodic quasi-phase-matching and control of the dispersion of the FF input pulses. Spectral shifts as high as $40 \mathrm{~nm}$ for input pulses with $70 \mathrm{~nm}$ spectral width are 
obtained at the end of the crystal together with high-quality bell-shaped spectral profiles, nearlytransform limited pulse duration and power penalty not exceeding $45 \%$.

We define the phase-mismatch between FF and SH pulses as $\Delta k(z)=2 k^{\omega}-k^{2 \omega}+2 \pi / \Lambda(z)$, with wave-numbers $k^{\omega}$ and $k^{2 \omega}$ depending on the material and on the wavelength, and with the poling period $\Lambda$ depending on the propagation distance $z$ in the case of aperiodic crystal poling. The magnitude of the shift and the shape of the FF output spectrum strongly depend not only on the poling period $^{11} \Lambda(\mathrm{z})$ but also on the chirp of the injected FF pulse $\mathrm{f}^{10,12}$. The present study is focused on the optimization of only the blue-shift effect, which is the most interesting one for the applications: in fact, i) it is accompanied by a temporal compression mechanism, helping to compensate temporal broadening induced by normal dispersion, and ii) it can not be obtained with alternative conventional techniques based on Raman amplification or Raman self-frequency-shift in optical fibers $^{13}$.

Experiments were performed using stoichiometric lithium tantalate (SLT) crystals, because of their large nonlinearity, high optical damage threshold and strong GVM. We used both conventional periodically poled SLT (PPSLT) and aperiodically poled SLT (aPPSLT) consisting of a sequence of uniform regions with different poling periods (HC Photonics Corp.). The FF pulses were derived from an optical parametric amplifier (OPA) pumped by an amplified Ti:sapphire laser system $(200 \mu \mathrm{J}, 60 \mathrm{fs}, 1 \mathrm{kHz})$. The OPA generates $70 \mathrm{~nm}$ nearly transform-limited pulses tunable in the near IR (1300-1500 nm) with widths around 40 fs (FWHM in intensity) and energy up to $5 \mu \mathrm{J}$. For the experiments, energies up to $900 \mathrm{~nJ}$ focused down to a spot-size of $111 \mu \mathrm{m}\left(1 / \mathrm{e}^{2}\right.$ half-width of the intensity profile) were used. The group delay dispersion (GDD) of the FF pulses was controlled by a double pass in a SF10 prism pair, which imparts a negative dispersion in this frequency range. The pulses at the output of the crystals were characterized by frequency-resolvedoptical gating (FROG). 
Preliminary experiments were performed in a conventional PPSLT crystal equipped with several poled regions with periods ranging from 17.7 to $18.5 \mu \mathrm{m}$, corresponding to $\Delta k$ values from -15000 to $-30000 \mathrm{~m}^{-1}$ at the adopted FF wavelength $(1420 \mathrm{~nm})$ and crystal temperature $\left(160^{\circ} \mathrm{C}\right)$. In a preliminary attempt to preserve the FF pulse-width at the output of the frequency shifter, the GDD of the FF pulses was chosen with an opposite sign with respect to that produced by the material ($2600 \mathrm{fs}^{2}$ ), so as to compensate for chromatic dispersion. At low energy, independently of phasemismatch, the FF output pulse spectrum is indistinguishable from the input one. At negative phasemismatch values, corresponding to a self-defocusing cascaded nonlinearity, at high enough intensity, spectral blue-shifts of the injected pulses were observed. The strongest blue-shifts were obtained for the poling period $\Lambda=18.1 \mu \mathrm{m}\left(\Delta \mathrm{k}=-23000 \mathrm{~m}^{-1}\right)$, and the corresponding spectra are reported in Fig. 1 (a). The spectral blue-shift of the FF pulses rapidly saturates upon increasing the pump energy. The spectra present prominent undesired red-tails with a peculiar bumpy behaviour, in agreement with the numerical simulations, performed in the plane-wave approximation ${ }^{12}$, reported in Fig. 1 (b). In the time domain, output pulse-widths as short as 39 fs were obtained at high energy, with modest pulse lengthening at lower energies.

In order to overcome the saturation of the spectral shift with input energy and to suppress the spectral red-tails, we considered the use of engineered aperiodically-poled nonlinear crystals, which offer a great flexibility in tailoring the local phase-mismatch along the propagation direction.. The design of the aperiodically-poled crystal was accomplished with an iterative genetic algorithm ${ }^{12}$, including, among the optimization parameters, also the chirp of the input FF pulse. The results obtained are reported in Fig. 2: panel (a) shows the piecewise poling period profile $\Lambda(z)$ and the corresponding space-dependent phase-mismatch $\Delta k(z)$, evaluated with respect to the input carrier wavelength. The values of $\Delta k(z)$ evolve in a linear fashion from -20000 to $20000 \mathrm{~m}^{-1}$. Figure 2 (a) indicates that the linear dependence is indeed the best choice and also identifies the best values of the initial and final mismatch. The optimized initial GGD value is $-1300 \mathrm{fs}^{2}$, which, for linear propagation, would result in a transform-limited pulse in the middle of the crystal. Figure 2 (b) 
shows the calculated FF pulse spectra at the output of the aPPLST crystal at different input energies. The spectral blue-shift of the FF pulses does not saturate upon increasing the pump energy and reaches a maximum value of $60 \mathrm{~nm}$ at $900 \mathrm{~nJ}$. The spectra do not present significant red-tails. Fig. 2 (c) shows the evolution of the FF spectrum during propagation in the nonlinear crystal, demonstrating that the spectrum drifts almost linearly with the propagation distance. This is at odds with the periodically-poled configuration, where the spectral drift occurs only in the first part of the crystal $^{10}$. Figure 2 (d) shows the FF input and output temporal intensity profiles. The temporal profile is smooth and does not exhibit breakup phenomena.

The experimental observed spectra at the output of the above described aPPSLT crystal are reported in Fig. 3 (a) for several input energies. Bell-shaped spectral profiles were found, with centres of mass almost coincident with the spectral peak for every FF input energy. The spectral shift no longer saturates upon increasing the input pulse energy, but, as far as cubic effects can be disregarded (which occurs below $900 \mathrm{~nJ}$ in the adopted experimental conditions), the spectral shift depends almost linearly on the FF energy, with a maximum amount of $40 \mathrm{~nm}$ for input pulses with a $70 \mathrm{~nm}$ bandwidth. Such a shift is lower than that predicted by numerical simulations (see Fig. 2 (b)), probably due to spatial effects not considered in the equations. In the temporal domain, nearly transform-limited output pulses with width below 65 fs were found even at relatively low intensities, where nonlinear compression mechanisms fail to be efficient. Figure 4 (a) shows a FROG trace acquired at full FF energy and Fig 4 (b) the retrieved temporal amplitude profile. It presents a slight asymmetric deformation on the leading edge, which is an evident signature of the quadratic self-steepening effect responsible for the shift. The pump depletion did not exceed a rather satisfactory value of $45 \%$. Since spectral shifts are strongly related to the strength of the nonlinear interaction, higher shifts could be in principle obtained with longer samples and/or different phase-mismatch profiles but at the price of higher pump depletion.

In conclusion, we have experimentally studied the use of aperiodically-poled nonlinear crystals to mould the spectral characteristics of broadband near-infrared femtosecond pulses. By optimizing 
the aperiodically poled structure and the dispersion of the input pulses, high quality blue-shifted spectra with carrier-wavelength tuning as high as $60 \%$ of the input spectral width were obtained with almost unaffected temporal properties and a tolerable power penalty of about $45 \%$. These results highlight the potential but also the limitations of frequency shifts induced by cascaded second order nonlinearities. In fact, both experiments and numerical simulations show that, even with optimized aperiodic structures, it is difficult to achieve clean spectral shifts larger than the pulse spectral width without a strong depletion of the pump field. 


\section{References}

1. G. I. Stegeman, R. Schiek, L. Torner, W. Torruellas, Y. Baek, D. Baboiu, Z. Wang, E. Van Stryland, D. J. Hagan, and G. Assanto, in Novel Optical Materials and Applications, I. C. Khoo, F. Simoni, and C. Umeton, eds. (Wiley, New York, 1997), Chap. 2, pp. 49-76

2. A. V. Buryak, P. Di Trapani, D. V. Skryabin, and S.Trillo, Phys. Rep. 370, 63 (2002).

3. S. Ashihara, T. Shimura, K. Kuroda, N. Ei Yu, S. Kurimura, K. Kitamura, M. Cha, and T. Taira, Appl. Phys. Lett. 84, 1055 (2004),

4. J. Moses and F. W. Wise, Opt. Lett. 31, 1881 (2006).

5. M. Marangoni, C. Manzoni, R. Ramponi, G. Cerullo, F. Baronio, C. De Angelis, and K. Kitamura, Opt. Lett. 31, 534 (2006)

6. J. Moses, and F. W. Wise, Phys. Rev. Lett., 97, 073903 (2006).

7. J. Moses, B. A. Malomed, and F. W. Wise, Phys. Rev. A 76, 021802(R) (2007).

8. F. Baronio, A. Barthélémy, S. Carrasco, V. Couderc, C. De Angelis, L. Lefort, Y. Min, P. H. Pioger, V. Quiring, L. Torner and W. Sohler, J. Opt. B 6, S182 (2004).

9. F. Ö. Ilday, K. Beckwitt, Y. F. Chen, H. Lim, and F. W. Wise, J. Opt. Soc. Am. B 21, 376 (2004).

10. F. Baronio, C. De Angelis, M. Marangoni, C. Manzoni, R. Ramponi, and G. Cerullo, Opt. Express 14, 4774 (2006).

11. K. Beckwitt, F. Ö. Ilday, and F. W. Wise, Opt. Lett. 29, 763 (2004).

12. M. Conforti, F. Baronio, C. De Angelis, G. Sanna, D. Pierleoni, and P. Bassi, Opt. Comm. 281, 1693 (2008).

13. Y. S. Kivshar, and G. P. Agrawal, Optical Solitons, $1^{\text {st }}$ edition (Academic Press, San Diego, California, 2003), pp. 93ff. 


\section{Captions}

Fig. 1: (a) Experimental FF spectra at the output of the PPSLT crystal for energy E=10 nJ (dashdotted line); $E=400 \mathrm{~nJ}$ (dashed line); $\mathrm{E}=900 \mathrm{~nJ}$ (solid line). (b) Simulated FF spectra at peak intensities $\mathrm{I}=\mathrm{xx} \mathrm{GW} / \mathrm{cm}^{2}$ (dash-dotted line); $\mathrm{I}=\mathrm{yy} \mathrm{GW} / \mathrm{cm}^{2}$ (dashed line); $\mathrm{I}=\mathrm{zz} \mathrm{GW} / \mathrm{cm}^{2}$ (solid line). The phase-mismatch is $\Delta k=-23000 \mathrm{~m}^{-1}(\Lambda=18.1 \mu \mathrm{m})$.

Fig. 2: (a) Poling period profiles $\Lambda(z)$ and effective mismatch profile $\Delta \mathrm{k}(z)$ of the engineered aperiodically-poled sample. (b) Numerical FF spectra at the output of the aPPSLT crystal: energy $\mathrm{I}=\mathrm{xx} \mathrm{GW} / \mathrm{cm}^{2}$ (dash-dotted line); $\mathrm{I}=\mathrm{yy} \mathrm{GW} / \mathrm{cm}^{2}$ (dashed line); $\mathrm{I}=\mathrm{zz} \mathrm{GW} / \mathrm{cm}^{2}$ (solid line). (c) FF spectral evolution in the $\lambda-z$ plane at $\mathrm{I}=\mathrm{zz} \mathrm{GW} / \mathrm{cm}^{2}$. (d) $F F$ input and output temporal intensity profile at $\mathrm{I}=\mathrm{zz} \mathrm{GW} / \mathrm{cm}^{2}$.

Fig.3: Experimental FF spectra at the output of the aperiodically-poled sample for several input pump energies: $\mathrm{E}=10 \mathrm{~nJ}$ (dash-dotted line); $\mathrm{E}=400 \mathrm{~nJ}$ (dashed line); $\mathrm{E}=900 \mathrm{~nJ}$ (solid line).

Fig. 4 (a) FROG trace of the FF output pulse for an input energy E=900 nJ and (b) corresponding amplitude profile of the pulse. 


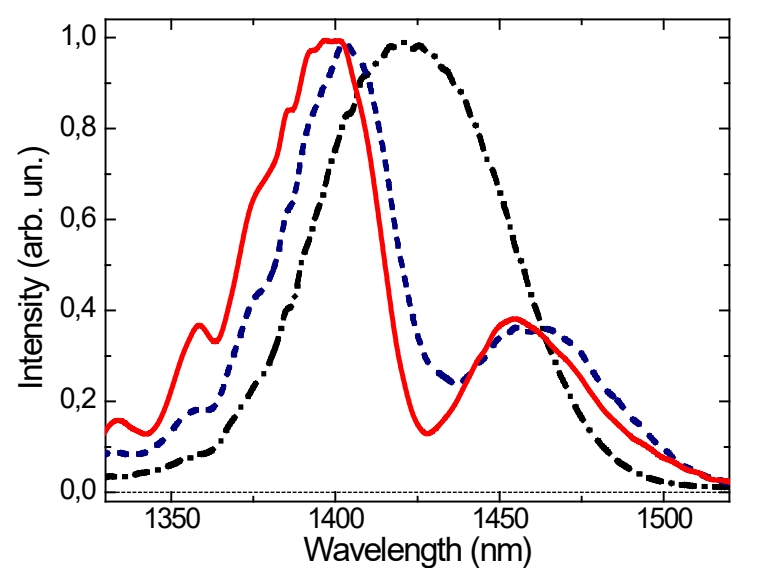

Fig. 1 (a)

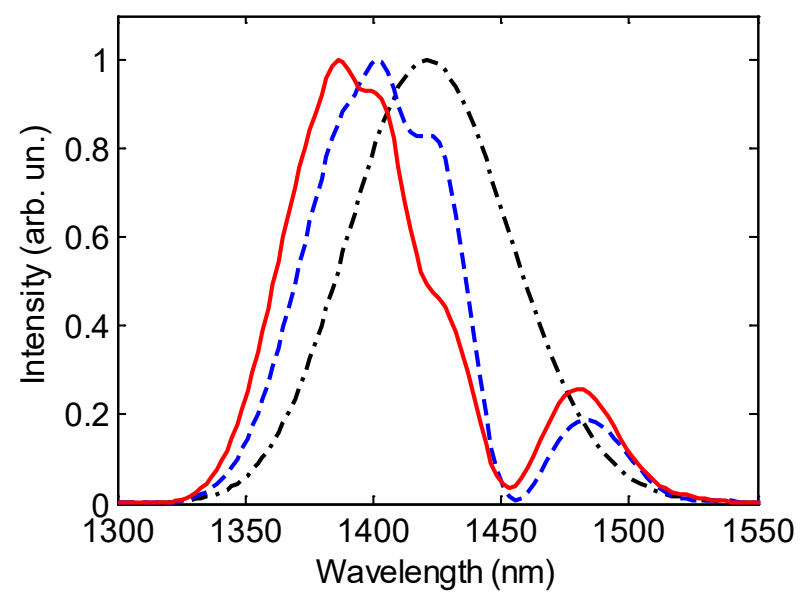

Fig. 1 (b)

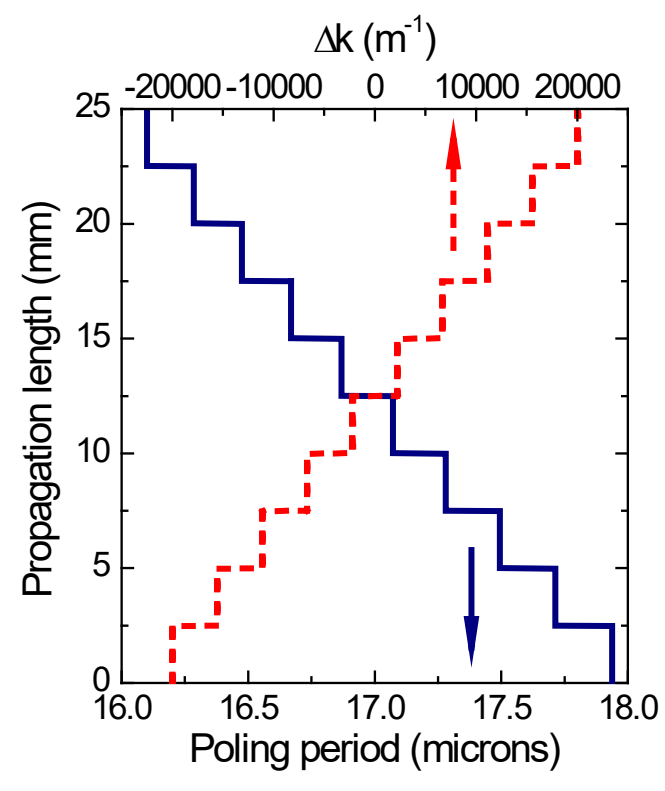

Fig. 2 (a)

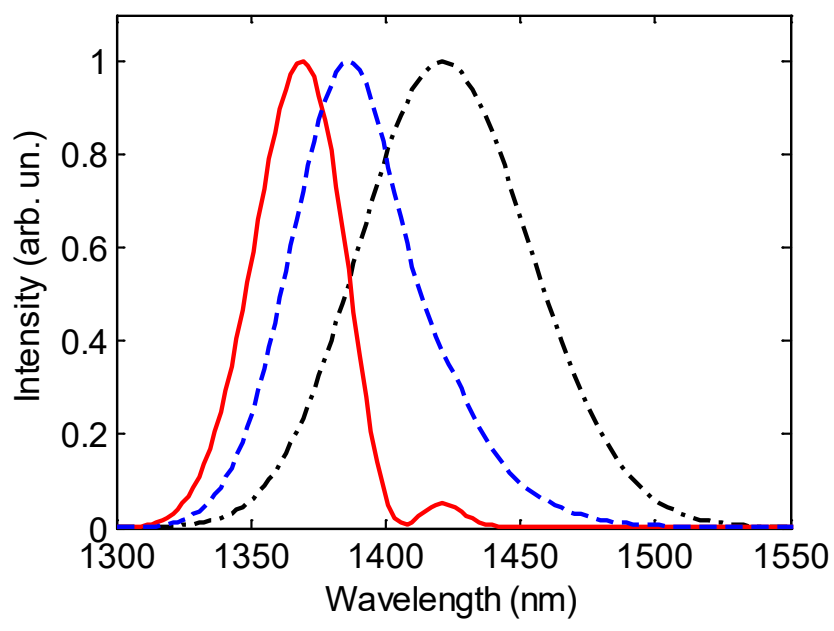

Fig. 2 (b) 


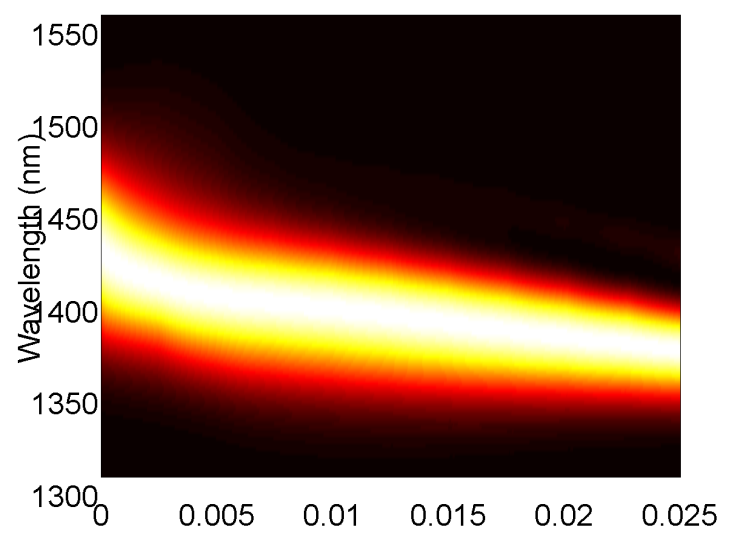

Fig. 2 (c)

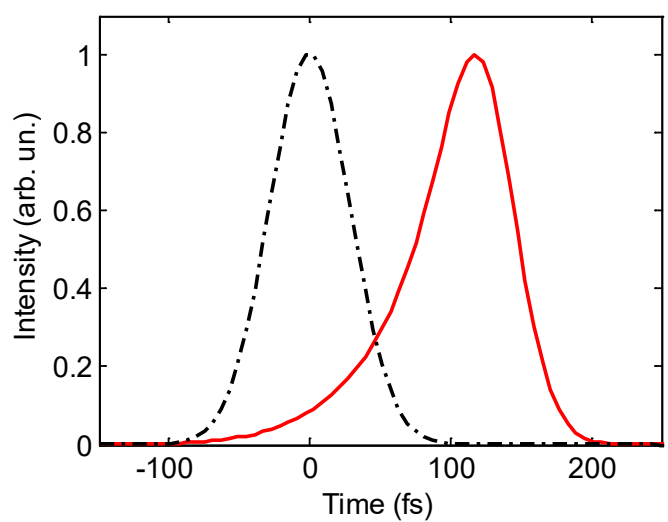

Fig. 2 (d)

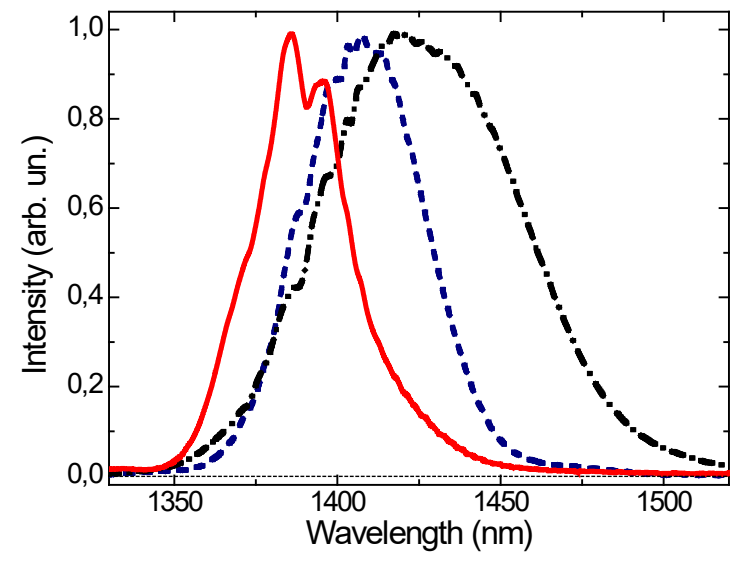

Fig. 3 


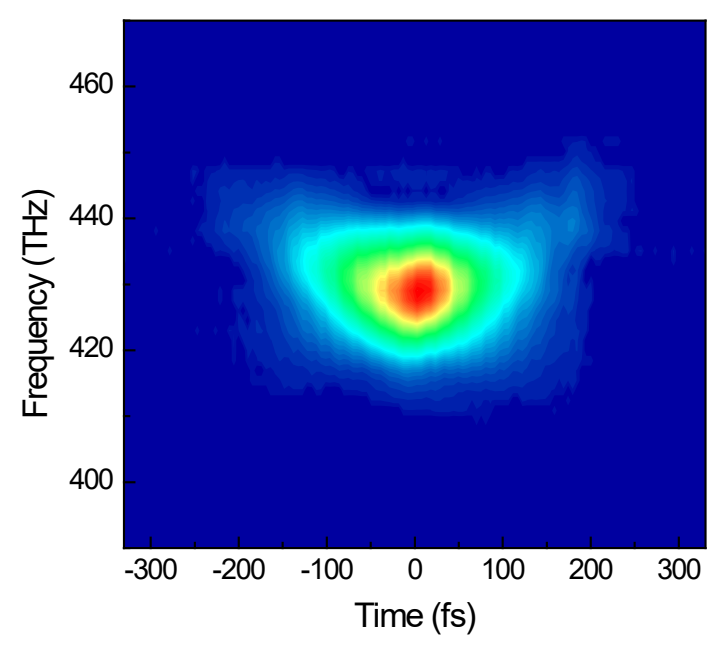

Fig. 4 (b)

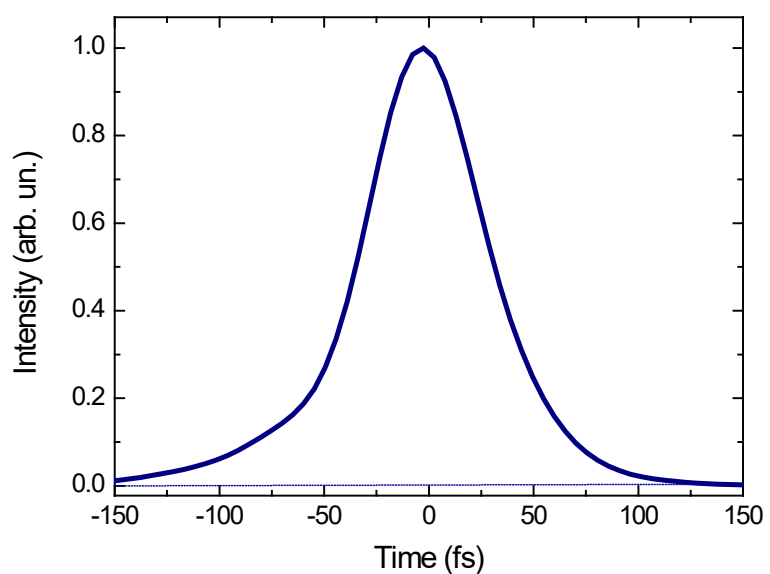

Fig. 4 (b) 